\title{
Methods of Measurement and Evaluation of Eye, Head and Shoulders Position in Neurological Practice
}

\author{
Patrik Kutilek ${ }^{1}$, Jiri Hozman ${ }^{1}$, Rudolf Cerny ${ }^{2}$ and Jan Hejda ${ }^{1}$ \\ ${ }^{1}$ Czech Technical University in Prague, Faculty of Biomedical Engineering \\ ${ }^{2}$ Charles University in Prague, Department of Neurology, 2nd Faculty of Medicine \\ Czech Republic
}

\section{Introduction}

The position of the eye, head and shoulders can be negatively influenced by many diseases of the nervous system, (particularly by visual and vestibular disorders) (Cerny R. et al, 2006). Disturbances of the cervical vertebral column are another frequent cause of abnormal head position. In this chapter we describe advanced methods of measuring the precise position of the eye, head and shoulders in space. The systems and methods are designed for use in neurology to discover relationships between some neurological disorders (such as disorders of vestibular system) and postural head alignment. We have designed a system and a set of procedures for evaluating the inclination (roll), flexion (pitch) and rotation (yaw) of the head and the inclination (roll) and rotation (yaw) of the shoulders with resolution and accuracy from $1^{\circ}$ to $2^{\circ}$ (Hozman et al, 2007). We will also deal with systems designed for parallel measurement of eye and head positions and a new portable system for studying eye and head movements at the same time is described as well (Charfreitag et al, 2008). The main goal of this study is to describe new systems and possibilities of the present methods determined for diagnostics and therapy support in clinical neurology. Furthermore, we describe the benefits of each method for diagnosis in neurology.

\section{Background and related works}

The measurement of eye position is an important diagnostic instrument in both clinical and experimental examination of human vestibular system (Cerny R. et al, 2006). Also, the simultaneous measurement of head (Murphy et al, 1991) and shoulders position (Raine et al, 1997) could contribute to better definition of diseases affecting the vestibular system (labyrinthine) function in man.

\subsection{Clinical significance of head posture measurement}

Abnormal head posture (AHP) is an important clinical sign of disease in many medical specialities. AHP is a consequence of dysfunction of musculoskeletal, visual and vestibular systems (Brandt et al, 2003). AHP is of particular importance in childhood, when 
developmental abnormalities of different origin can manifest with AHP as a main clinical symptom. The differential diagnosis is broad and quantitative assessment of head position in space it is important for both treatment and evaluation of disease evolution. In an Italian study 73 children referred by paediatricians the most common cause of AHP was orthopaedic disease (congenital muscular torticollis, 35 cases) followed by ocular motor palsy (mostly superior oblique palsy, 25 cases). Neurological disease was found in 5 cases, in 8 cases no underlying disease was indentified (Nucci et al, 2005).

Most peculiar forms of AHP are due to cervical dystonia, a movement disorder due to the disturbance of motor control of cervical muscles. Exact pathophysiology of this disabling and hard to treat condition is not known and includes local, suprasegmental and psychological factors. It can be classified according to the abnormal positioning of the head and spine into ante/retrocollis (sagittal plane), laterocollis (frontal plane) and rotatocollis (horizontal plane), pure forms are rare, typical is combination (torticollis). The pattern of muscles involved in generation of the AHP can be inferred from the head position. Objective and quantitative measurement of head position is of great importance, as treatment with botulotoxin (nowadays first choice) requires exact identification of muscles involved in AHP generation and follow up of treatment efficacy with objective head positions recordings is important for choosing optimal long term treatment strategy. Standard assessment scales for torticollis use semiquantative clinical scores or simple goniometers with low precision (Galardi et al, 2003; Novak et al, 2010).

Blockades and disease of cervical spine due to spondylosis or trauma are very common cause of AHP in clinical practice. Here the quantitative head posture measurement is not imperative, but simple objective recording of abnormality evolution can be useful in chronic cases and when cervical spine surgery is considered.

AHP is a frequent and important sign in ophthalmology, particularly in childhood. It represents compensation of abnormal eye position and/or motility. Paralyses of eye muscles are compensated by a tilt of the head in direction of the weakened muscle. In congenital nystagmus the AHP tends to shift gaze direction in the null zone of the nystagmus. As a result of the compensatory head position, the vision acuity is enhanced or restored, but unbalanced muscle activation can lead to cervical spine disorders in the long term. Surgical procedures aimed at correction of the eyeball position are effective in repairing the AHP and are considered treatment of choice (artificial divergence, Kestenbaum surgery). The dosage of ocular muscle retroposition/resection depends on the angle of AHP with fixation of distant target. The reduction of abnormal head turn with $1 \mathrm{~mm}$ muscle resection was $1.4^{\circ}$ head turn on average in one study (Gräf et al, 2001).

Ocular tilt reaction is a well established symptom of dysfunction of the graviceptive pathways starting from the otholithic maculae of the inner ear to the vestibular nuclei and paramedian thalamus. This syndrome is defined by the triad of signs - head tilt, ocular globe rotation a deviation of the subjective visual vertical. All deviations directs towards the weak labyrinth, or to the contralateral side after crossing at the pontine level, in the case of brain stem lesions. Head tilt in the frontal plane is usually quickly compensated, after the acute phase is over, but more subtle signs (ocular rotation and subjective vertical) can last for weeks and months. Horizontal eyes alignment is precisely regulated within narrow range of several degrees (Halmagyi et al, 1991), (Brandt \& Dieterich, 1994). Deviations in the 
horizontal plane are also easily appreciated even by naked eye during examination. Little is known about head turn in vestibular syndromes. This type of deviation is hard to assess by observation only, indeed, only gross deviations in cases of ocular torticollis are used in clinical practice and regularly cited in literature. Vestibular imbalance due to unilateral labyrinthine failure causes vestibulospinal deviations towards the weaker labyrinth (Hautant reaction, Romberg deviation in standing with closed eyes etc.). It is reasonable to expect head turns of several degrees due to the functional imbalance between the activity horizontal channels. In contrast to the tilt reaction such a finding was not well described until now. Probably, this type of vestibular rotatocollis is compensated by spatial visual clues with open eyes and can be easily overlooked. In this situation, precise technique for head rotation measurement would be of paramount importance.

Last, but not least, precise 3D head position measurement has many potential implications for physical medicine and rehabilitation, particularly in the management and diagnosis of disorders affecting cervical spine. Head position in the sagittal plane is very variable and influenced by many factors, particularly habitual holding of the spine as a whole. Habitual head anteflexion with chronic overload of cervical and upper thoracic spine and muscle imbalance is typical consequence of uncompensated sedentary way of life, starting already in school age. Main reference for sagittal plane is so called Frankfort horizontal (line connecting meatus acusticus with the orbital floor or line connecting tragus with the outer eye canthus), see Figure 1. In most subjects this line is inclined forward bellow the space horizontal, in the extensor type of cervical positions is reclined backwards. The real position of Frankfort horizontal can vary more than $20^{\circ}$ in the normative population, in comparison, the position of the eyes in frontal plane is held tightly within several degrees only (Harrison \& Wojtowicz, 1996).

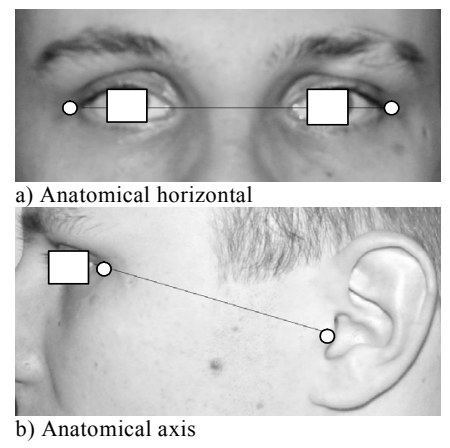

Fig. 1. Anatomical Frankfort horizontal and axis.

Precise measurement of head position in rehabilitation and physical medicine is important not only for objective diagnosis of the cervical spine abnormalities, but also as a means of cervical kinesthesia assessment. In this test the ability of the tested subject to assume exact position in space without visual clues is examined (Palmgren et al, 2009). Normal subjects are able to attain desired position with precision of several degrees. Again, these differences are below the discrimination capacity of simple observation or protractor measurement. It is hypothesized, that abnormal setting of cervical proprioception can play important role in many conditions like whiplash injury syndrome, chronic tension headache, cervicogennic 
vertigo, anteflexion headache etc. (Raine \& Twomey, 1997). Evidence of abnormal cervical proprioception would be an important step in better understanding of these common clinical problems.

\subsection{Monitoring head and shoulders movements}

At present, an orthopedic goniometer is the widely used and standard way to simply and rapidly measure angles in clinical practice. However, there are some limitations, especially in case of head and shoulder posture measurement. Due to the combination of three movement components (in the three dimensional space), the measurement using only one goniometer is clearly insufficient. The following overview serves as enumeration of the applications related to the technology available during the last years. This enumeration is not exhaustive but the most important works in the area are included. The methods are typical by using some tools or technology.

Young, 1988, designeda new method to study head position by mirrors. The main principle of new approach is based on using three mirrors and special head markers. The resulting images are taken by one camera. After this, a set of vertical or horizontal lines is drawn with respect to the reference points i.e. markers. The last step is measurement of the relevant angles by a protractor. Head tilt (inclination), head turn (rotation) and chin elevation or depression (flexion/extension) is evaluated. One drawback is the evaluation method based on vertical or horizontal lines defined by reference points, i.e. markers and thus wide variation in cranial configuration found between patients and associated with age.

Murphy et al, 1991, described a system for measuring and recording cranial posture in a dynamic manner. Measurement of the declination and inclination was performed by inclinometers. Inclinometers are widely used instruments for measuring angles of elevation or inclination of an object with respect to gravity based on the accelerometers. Inclinometer was attached to the spectacle rims. Processing of the inclinometer voltages was performed by a modified universal data logger. The inclinometer was calibrated by plastic visor and a perpendicular spirit level. However, principle of the inclinometer does not provide measurement of head rotation.

Ferrario et al, 1994, integrated a method based on the photographic technique, radiographic technique, cephalometric measurements and photographic measurements. The measured subjects were photographed and X-rayed in the same room. The set of standardized marks was traced on all the records. On all photographs, the soft tissues were traced, and the angle between the soft tissue marks and true vertical was calculated. The same angle was calculated on the cephalometric films, and the difference between the two measurements was used to compute the position of the soft and hard tissues. These new values were compared with the values previously observed. The main drawback is exposition of patients to X-ray and relatively time consuming procedures.

Ferrario et al, 1995, developed a new method based on television technology that was faster than conventional analysis. Subject's body and face were identified by 12 points. All subjects were pictured using a standardized technique for frontal views of the total body and lateral views of the neck and face. After 20 seconds of standing, two 2-second films were taken of each subject. On the basis of an image analysis program, the specified angles were calculated after digitizing the recorded films. 
Galardi et al, 2003, developed an objective method for measuring posture and voluntary movements in patients with cervical dystonia using Fastrack. Fastrack is commercial widely used electromagnetic system consisting of a stationary transmitter station and four sensors placed on patient's head. The head position in space was reconstructed based on sensor signals and exact values of angles were observed from the axial, sagittal and coronal planes. The drawback is its inaccuracy in determining the exact position in space because of relatively large sensors placed on the patient's head and therefore inaccurate determination of the anatomical axes. Second drawback is the negatively affected accuracy of an electromagnetic system by other laboratory systems.

Hozman et al, 2004, proposed a new method based on the application of three digital cameras placed on a stand and appropriate image processing software. The method was designed for use in neurology to discover relationships between some neurological disorders (such as disorders of vestibular system) and postural head alignment. The objective was to develop a technique for precise head posture measurement or, in other words, for measuring the native position of the head in 3D space. The technique was aimed at determining differences between the anatomical coordinate system (ACS) and the physical coordinate system (PCS). Pictures of the head marked on tragus and outer eye canthus are taken simultaneously by three digital cameras aligned by laser beam. Head position was measured with precision of $0.5^{\circ}$ in three planes (rotation-yaw, flexion-pitch and inclination-roll). Hozman et al, 2005, described the new modified system and results are shown and measured on normal subjects. The disadvantage is complicated calibration and the impossibility of a frontal view of the measured subject (Hozman et al, 2007).

Cerny et al, 2006, described second advanced generation of the system. Head position was measured with precision of $0.5^{\circ}$ in three planes. Mean values of the head position (100 healthy controls) are : retro flexion $21.7^{\circ}$; inclination to the right $0.2^{\circ}$; head rotation to the left $1.7^{\circ}$.

Meers et al, 2008, developed accurate methods for pinpointing the position of infrared LEDs using an inexpensive USB camera and low-cost algorithms for estimating the 3D coordinates of the LEDs based on known geometry. LEDs are implemented in the frame of eye-glasses. The system is accurate low-cost head-pose tracking system. Experimental results are provided demonstrating a head pose tracking accuracy of less than $0.5^{\circ}$ when the user is within one meter from the camera. However, the system does not define the anatomical axis of the head and the adaptation of the system is impossible for measurement of anatomical angles.

Recently a number of instruments and tools based on commercial systems have been developed for evaluating the position of the head and shoulders. An example is Zebris motion analysis system (zebris Medical $\mathrm{GmbH}$ ). Special instruments primarily allow studying ranges of motion of the head, ranges of motion of the spine and coordination of movement. The modified Zebris also allows studying the movement of the jaw. Zebris detects small misalignment of the lower jaw. The three-dimensional measuring coordinates of the ultrasonic markers can be recorded with an overall scanning rate of 200 measurements per second. The modified system consists of a face bow with integrated receiver module and an optimally balanced mandible which measures sensor close to the mandible joint. Unfortunately, the system also does not define the anatomical axis of the head and the adaptation of the system is complicated. 
There are other modified commercial diagnostic systems based on ultrasonic measurement method (sonoSens Monitor), a camera method (Vicon motion systems, LUKOtronic AS100/AS200), a gyro-accelerometer sensors (Xsens motion trackers), etc. But the systems have the similar disadvantages such as complex preparation, very large sensors or the inability to accurately define the anatomical coordinate system.

\subsection{Monitoring eye and head movements}

Monitoring eye movements and plotting their trajectories have a long tradition in medical practice. The measurement of eye position is an important examination tool in understanding human vestibular system. It is used as a diagnostic tool in neurology and psychology (Brandt et al, 2003). Eye tracking is a widely used method of measuring the point of gaze or the motion of an eye relative to the head. An eye tracker is a device for measuring eye position and movement. There are number of methods of measuring eye movement. Eye trackers fall into three categories:

One type uses an attachment to the eye, such as a special contact lens with an embedded mirror or magnetic field sensor. Measurements with contact lenses have provided extremely sensitive recordings of eye movement. However, mechanical elements attached to the eye can negatively influence patient's eye.

The second category uses electric potentials measured by electrodes placed around the eyes. The eyes are the origin of a steady electric potential field. The electric signal that can be derived using two pairs of contact electrodes placed on the skin around eye is called Electrooculogram (EOG). This EOG is sensitive to the saccadic spike potentials from the ocular muscles. The electric potential field can also be detected in total darkness and if the eyes are closed.

The third category uses non-contact, optical method for measuring eye motion. The method is called Videooculography (VOG). Optical methods are widely used for gaze tracking and are favoured for being non-invasive and inexpensive. By looking to the eye we can see its elements - outer filamentous layer with title sclera, further is cornea, iris and eye pupilla. Light, typically infrared, is reflected from the eye and sensed by a camera. Video based eye trackers usually use the corneal reflection and the centre of the pupil as subjects to track over time. The videooculography based on IR spectrum usually uses the infrared light created by a LED (light emitting diode) diode with a wavelength approximately $\lambda=880$ 940nm. The VOG method in the IR spectrum detects the pupil using an appropriate light that makes it completely black. The Advantage of this method is relatively easy pupil detection and good quality reflection, most often using an IR LED diode. Disadvantage and limitation is a need to make measurements without access of visible light, i.e. in conditions that do not correspond with patient's real situation.

Eye analysis in the visible light spectrum is far more complicated. The method is called passive, because the eye is scanned in the visible light spectrum due to the diffused visible light. The method without the IR supplementary light is not only safer for the patient (undesirably warms up the eye), but also much more preferable, because it does not necessarily need suppression of background light. Detection can be done due to the sclera and iris interface. Disadvantages of these methods are the uncontrolled lighting from scattered sources, considerable luminous artefacts and high computational power. Also 
accuracy of these methods is rather poor, because in contrast to the pupil of the eye which is visible during measuring, the interface between sclera and iris is often hidden.

For parallel measurement of head and eye position (Eui et al, 2007) the best way is to use a VOG method that is based on the principle of scanning the eye (Ruian et al, 2006) using a mobile set of video cameras and consequent data post-processing to a different result in IR (infra red) or visible light spectrum (e.g. nystagmogram, fixing the eye to the projected area etc.). The mobile set is then attached to the head position measurement system based, for example, on gyro-accelerometer sensors. This new parallel measurement method has not been systematically studied and bothmentioned measurement methods have been examined only separately.

\section{Precise advanced eye, head and shoulders position measurement}

Numerous systems for evaluation of eye and upper body parts positions are currently offered on the market, but their wider application is impeded by high financial demands and inaccuracy, because these universal systems are not usually designed for application to study a particular body part - head and shoulders. In the following part of the chapter we will describe the specialized systems designed at CTU Prague and the other labs, to precisely measure the eye, head and shoulders posture at the same time.

\subsection{Precise head and shoulders posture measurement}

Our new non-invasive head position measurement method was designed for use in neurology to discover relationships between some neurological disorders and postural alignment. The objective was to develop a technique for precise and non-invasive head posture measurement in $3 \mathrm{D}$ space. The technique is aimed at determining differences between the anatomical coordinate system and the physical coordinate system with accuracy from one to two degrees for inclination and rotation (Harrison \& Wojtowicz, 1996). Pictures or recordings of the head marked on the tragus and the outer eye canthus, see Figure 1, and the shoulders marked on acromions are taken simultaneously by cameras aligned by a laser beam, magnetometers or inertial systems.

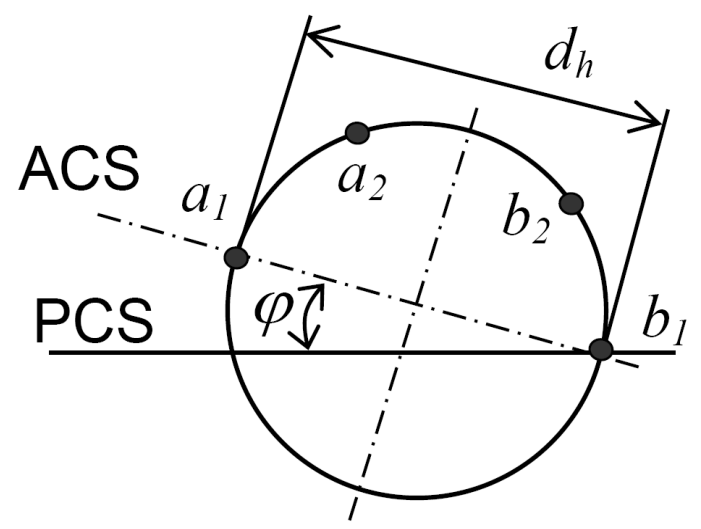

Fig. 2. Geometry used for measuring the head position (top view). 
In our recent method for studying only head position (Kutilek \& Hozman, 2009), only two cameras are required for determining head positions i.e. anatomical coordinate system in the physical coordinate system. The rotation and inclination of the head is calculated from the difference between the tragus coordinates in the left-profile and right-profile image or eventually in the frontal picture and top view picture of head. The coordinates of the left and right tragus are evaluated by finding the centre of the rounded mark attached to the tragus. After evaluating the coordinates of the tragus in the captured images, the angles of head rotation and inclination are calculated. The images are captured simultaneously, using two cameras, which are calibrated. The adjustment of the camera system defines the position of the head in the instrument coordinate system. The position of the head in the physical coordinate system is determined by special software and the exactly known adjustment of the camera system.

Generally, the angles of rotation and inclination can be determined by vector $v$ and vector $u$, which represent the coordinates of the points evaluated in the image, see Figure 2. The angle (for example rotation $\varphi$ ) is calculated as follows (1):

$$
\varphi=\operatorname{arctg}\left(\frac{a_{1 y}[p x]-b_{1 y}[p x]}{a_{1 x}[p x]-b_{1 x}[p x]}\right)
$$

where

$$
\begin{aligned}
& u=\left(a_{1 x}[p x], a_{1 y}[p x]\right), \\
& v=\left(b_{1 x}[p x], b_{1 y}[p x]\right) .
\end{aligned}
$$

The $a_{1 x}$ is the x-axis coordinate and $a_{1 y}$ is the y-axis coordinate of the left tragus in the top view image. The $b_{1 x}$ is the $\mathrm{x}$-axis coordinate and $b_{1 y}$ is the $\mathrm{y}$-axis coordinate of the right tragus in top view image. We can determine the angle of inclination $\sigma$ in the similar way in the frontal view image. After calculation of the angles in the instrument coordinate system, the angles in physical coordinate system are derived by mathematical transformation.

If we use profile photographs (side shots) and want to evaluate the elevation/depression of the head in the instrument coordinate system and sagittal plane, it is also a mathematically simple problem. The flexion value is measured relatively as the inclination of the connecting line between the coordinates of tragus and the exterior eye corner. The coordinates are evaluated by finding the centre of the rounded mark attached to the tragus and outer eye canthus of the patient. The angle between the anatomical and physical or instrument horizontal (depends on adjustment of instrument) is determined by the angle between vector $v$ (horizontal vector), here given by the camera position, and vector $u$, which here represents the coordinates of the points (corresponding to the tragus marker and exterior eye corner marker) evaluated in the image. The angle is calculated as follows (4):

$$
\omega=\arccos \left(\frac{\vec{u} \cdot \vec{v}}{|\vec{u}| \cdot|\vec{v}|}\right)=\arccos \left(\frac{u_{x} \cdot v_{x}+u_{y} \cdot v_{y}}{\sqrt{u_{x}^{2}+u_{y}^{2}} \cdot \sqrt{v_{x}^{2}+v_{y}^{2}}}\right)
$$


where

$$
\begin{gathered}
u=\left(a_{1 x}[p x]-a_{2 x}[p x], a_{1 y}[p x]-a_{2 y}[p x]\right), \\
v=(1,0) .
\end{gathered}
$$

$a_{1 x}$ is the x-axis coordinate and $a_{1 y}$ is the y-axis coordinate of the tragus in the profile image, and $a_{2 x}$ is the x-axis coordinate and $a_{2 y}$ is the y-axis coordinate of the outer eye canthus in the profile image.

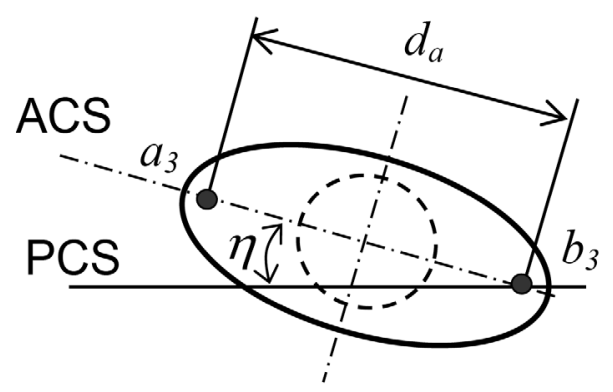

Fig. 3. Geometry used for measuring the shoulder position (top view).

For evaluating shoulder inclination and shoulder rotation, we use a method similar to the method that we use for evaluating head position. Picture of acromions on shoulders is taken by cameras. A medical doctor indicates acromions with marks for easy location of these anatomical points in the pictures. If the clinical investigation is carried out by an experienced medical doctor, it is not necessary to apply coloured marks to the anatomical parts of the body before making an examination using camera system. The software can also determine the angular displacement of the head to the shoulders for rotation, using the formula

$$
\kappa=\eta-\varphi
$$

where the $\eta$ is angle of shoulders rotation (Figure 3) and $\varphi$ is angle of head rotation (Figure 2). The angular displacement of the head to the shoulders for inclination

$$
\lambda=\zeta-\sigma
$$

where the $\zeta$ is angle of shoulders inclination and $\sigma$ is angle of head inclination.

In the way described above, based on identifying anatomical points with the use of two cameras (Figure 4) we can avoid influencing patients while we are measuring the inclination (roll), flexion (pitch) and rotation (yaw) of the head and shoulders.

The last proposed system is a combination of camera and gyroscope-accelerometer system. One camera is placed vertically above the patient's head. The second camera is positioned behind the patient in order not to impede the frontal view of the patient during the examination. Shoulders are marked in 3D space by a two IR diodes. Position of the two anatomical points (acromions) on the shoulders is defined by the two IR diodes. Diodes are placed on patient's shoulders by the physician before the examination. The position of 
head's anatomical coordinate system is defined by a special instrument, see Figure 5 . A physician puts the instrument on patient's head. The physician defines the position of the anatomical axes of the head by a special ruler on the instrument. Position of the head is defined by the two IR light diodes mounted on the instrument and gyroscope-accelerometer system. Rotation and inclination is then determined from the position of two infrared diodes mounted on the instrument and by two cameras. Flexion/extension is determined by accurate gyroscope-accelerometer system made by the company TRIVISIO, which is also part of the special instrument. The proposed special instrument is in Figure 5.

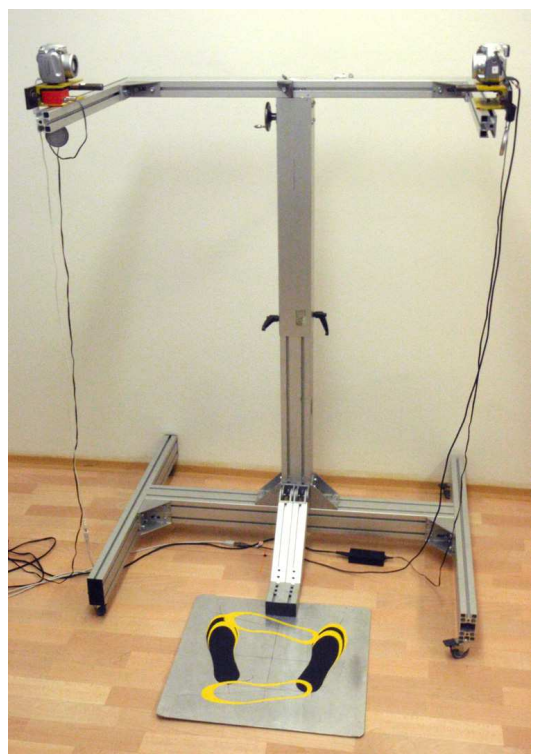

Fig. 4. The two-arm stand with fixed two cameras and laser collimators designed for the precise adjustment of the system.

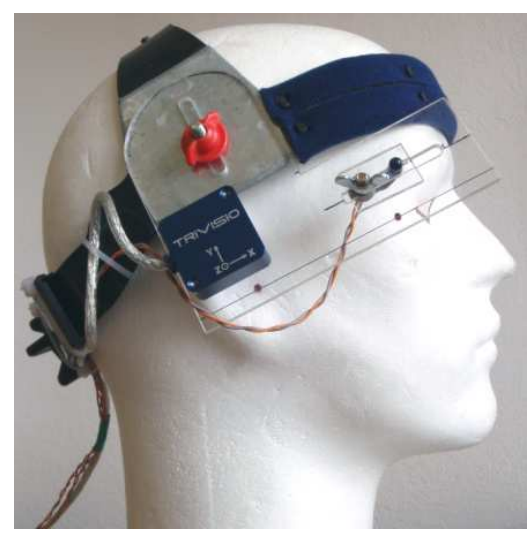

Fig. 5. Combined inertial system with infrared marker for detecting the position by IR camera system. 


\subsection{Measurement of eye and head position}

Despite the fact that an accurate method for measuring the head position and the eye position could contribute to diagnosis of vestibular system, this issue has not been systematically studied (Hozman et al, 2008).

Horizontal and vertical eye movements can be measured by an image of the eye (Moore et al, 2006) by detecting the edges of the pupil (iris) and fitting them into an ellipse (Li, 2006). The main aim of the analysis of eye movements is to obtain the centre of the pupil or the iris. The torsion measurement needs high quality iris description. The video system PAL (NTSC) record video with frequency $50 \mathrm{~Hz}(60 \mathrm{~Hz})$ non-interlace. These video systems are too slow to capture images of the eye movements, e.g. torsion iris description. In medical practice documented eye movements were with frequency approximately 200-250 Hz. These movements present angular change approximately $400-450 \%$ s.

We used the detection method which searches interface points between the pupil and the iris or between the iris and the sclera. The points are base of the mathematical function (e.g. circle or ellipse). Goal of our solution is to use eye movements' detection in comparison with the stimulation scene. The scene can be showed on the LCD screen or through the special HMD display unit in 2D or 3D space.

We used a new system based on finding the outline pupil of the eye. We applied modified Starburst (Duchowski et al, 2009) algorithm (Ruian et al, 2006), which we used in the IR spectrum or in the visible spectrum. The system and algorithm was first published by Iowa University in 2006 (Li, 2006). Thanks to the special 3D HMD projection displays we used Starburst algorithm for measuring in the IR spectrum and appropriate LED diode to illuminate the eye. The method is called active, because the eye is scanned in the infra red spectrum. Goal of eye movements' measurement was location of the centre of the pupil area. Method of finding the margins of the pupil (IR spectrum) or the iris (visible spectrum) is limited by quantity of the rays. The starting point shoots the rays to generate candidate pupil points. The candidate pupil points shoot rays back towards the start point to detect more candidate pupil points. This two-stage detection method takes advantage of the elliptical profile of the pupil contour to preferentially detect features on the pupil contour.

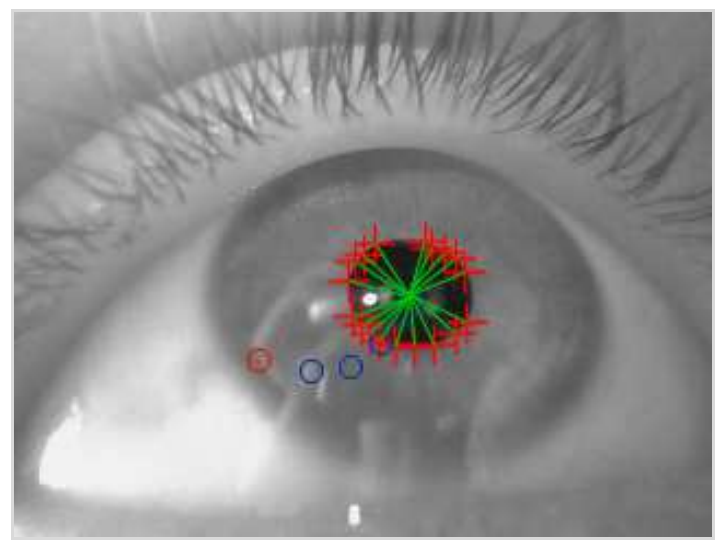

Fig. 6. Location of the pupil center with the rays. 
The circle (see Figure 6) shows the centre of the pupil after the second iteration (and after the next iteration) and includes determined points. The iteration to find the centre of the pupil was stopped when the detected centre of the new points turned less than $d=10$ pixels. Thanks to exponential calculation the error of the pupil centre is about \pm 10 pixels of the whole circle bearings and it is important from the point of view of the found points' fit resulting ellipse. At the end, we can find centre of the pupil from the resulting ellipse.

There are more possible methods how we can put together the resulting ellipse. We chose method (Ruian et al, 2006) Random Sample Consensus (RANSAC) to solve the problem with large error points (Lee \& Park, 2009). The method RANSAC is an efficient technique for completion of the model in the presence of large, but unknown percentage outlines in sample measurement. In our case all internal found points are probable points that correspond with outline of the pupil. RANSAC algorithm was used to estimate parameters of the mathematical model from a set of observed data which contains outliers. On the basis of the MATLAB documentation we used optimal mathematical model to create the ellipse Nelder-Mead's algorithm.

For the eye stimulation we used commercial HMD system eMagin Z800 3DVisor personal display with integrated head tracker which can measure head position in the 3D space. The Z800 3DVisor is the personal display system to combine two OLED (organic light-emitting diode) micro displays with stereovision 3D capabilities. Stereo vision refers to the human ability to see in three dimensions and most often refers to depth perception (the ability to determine the approximate distance of objects). Stereovision 3D provides this experience by delivering two distinct images simultaneously on two separate screens, one for each eye. The Z800 3DVisor personal display is used to stimulate the eye in 2D or 3D space. The position of eye and the position of head can be recorded simultaneously by the video camera and integrated head tracker to the laptop.

The HMD displays eMagin Z800 3DVisor's integrated head tracker uses MEMS (micro-electromechanical system) accelerometers and gyroscopes to detect motion. The head tracker features three gyroscopes, one each for the $\mathrm{x}_{-}, \mathrm{y}_{-}^{-}$, and $\mathrm{z}$-axis. In addition, the head tracker contains corresponding compasses and accelerometers to ensure performance over varying forms of motion. Such equipment has not been used before in medical practice. From the point of view of contemporary technology there is a possibility to use more accurate miniature 3D inertial measurement unit/motion sensors (IMU) with accelerometer, magnetometer and gyroscope (for example Xsens motion technologies) and custom made Head Mounted Display (HMD). We used the head tracker for the measurement of head position.

For acquisition of the head motion we programmed special software based on Z800 3DVisor SDK 2.2. The software retrieves position of the head from the build-in head tracker through the USB connection and saves the measured results in to the CSV (comma-separated values) file. The result of measurement can be presented graphically as a graph of the head position. By this set we are able to measure eye and head movements continuously and simultaneously.

During the measurement problematic parts had to be solved before the next biomedical tests. The problems were with weight, sharp edges on the semi-permeable mirrors and minimal place between personal display Z800 3DVisor and the eyes. 
On the previous base type we made the new projection displays, which allowed tracing projection on the LCD monitor or the projection screen in visible spectrum. Detection algorithm can measure the eye position and the scene position. The video files of positions are merged into the date file. The second version of projection displays has lower weight, does not contain any sharp edges and includes the cameras, which are connected with the help of USB (Universal Serial Bus) interface and does not use any special recorder. Power supply is solved over the USB port. We used record software TVideoGrabber. The software TVideoGrabber can set capture parameters (30 FPS - Frames Per Second, 640 x 480 pixels, RGB24, data format AVI). We used an external flash from photographic apparatus for synchronisation between two cameras (In the future we will use the TVideoGrabber component with more threads. The threads will start recording from several video sources at the same time).

The next type of our projection displays is designated for measuring in the IR spectrum. The third projection displays use the IR USB cameras which record eye movements. The type of these projection displays must use cameras which support eye movements scans in the IR spectrum because lighting is already poor. This type of projection displays combines a unique system for measuring eye movements and head position with $2 \mathrm{D}$ or 3D stimulation.

The specialized glasses - projection displays for neurological examination can be used without the LCD monitor thanks to build-in HMD 3D projections displays eMagin Z800 3DVisor. These projections displays can be used as a mobile system as well.. We use experimental systems for monitoring eye movements with different luminous conditions (in the visible spectrum or in the IR spectrum) or using different stimulation sources (e.g. record eye movements at specific activities - „eye Holter", long time eye movements record, 2D and 3D stimulation et al.).

\section{Interpretation and evaluation of eye, head and shoulders position}

For analysis of the positions we use simple methods based on combination of the basic methods of evaluation of individual body parts. Below we describe methods for accurate interpretation of the measured data. Given that the systems provide the processed data, the assessment is simple for physicians. Physicians only need to observe the conditions of measurement, such as the precise adjustment of the system before the measurement. They also need to respect the maximum certified accuracy of systems.

\subsection{Evaluation of head and shoulders position}

It is mathematically a simple problem to determine the inclination, rotation and flexion/extension of the head from photographs and by gyro-accelerometer sensor. The angle values are measured and transformed automatically to physical coordinate system. The measurement process is usually carried out according to a predefined procedure to be followed, see Figure 7. The process is based on two main steps/parts of measurement and computational algorithm. First part of algorithm is designed for the precise adjustment of the system. The second part is intended to measure patient's body segments and to calculate of the angles in physical coordinate system. 


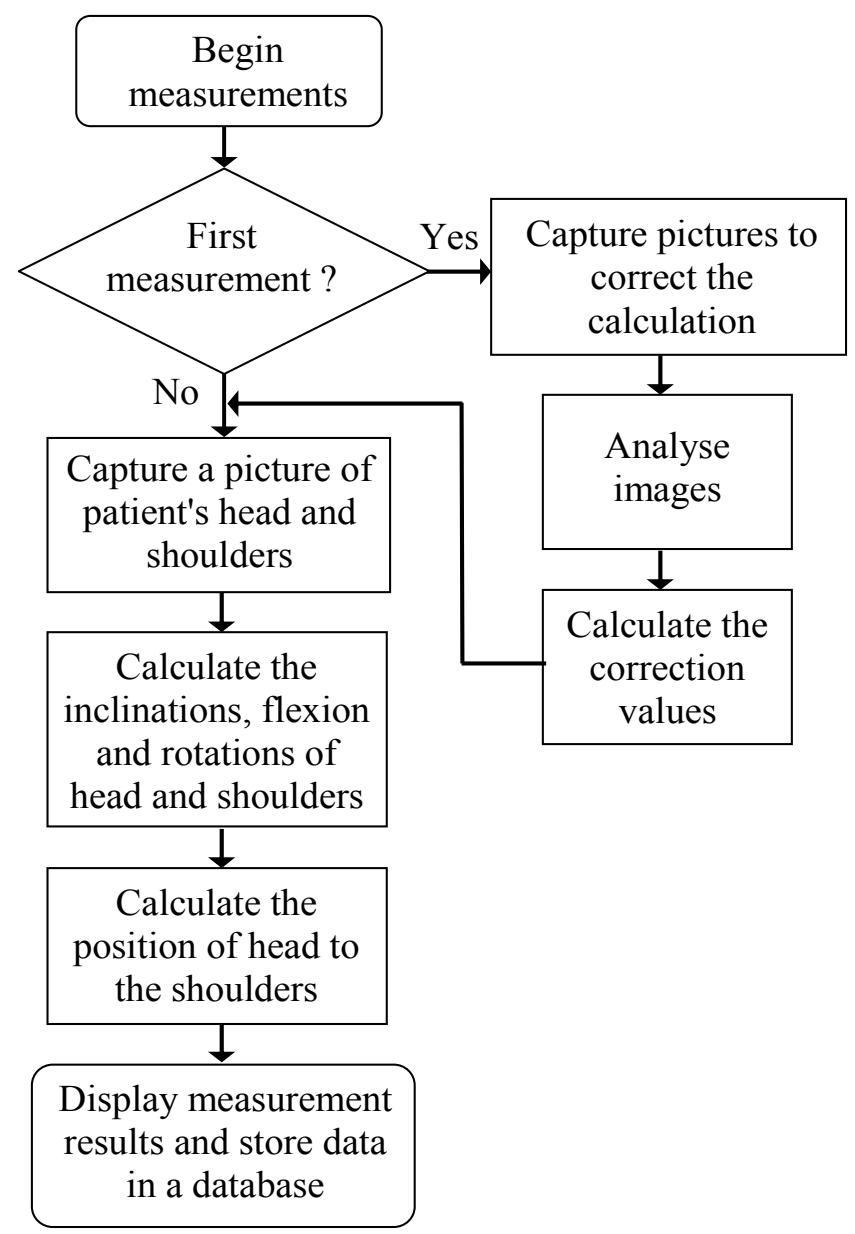

Fig. 7. Flowchart of clinical measurements using designed camera system.

The described systems provide direct information for physicians on the current position of the patient's head and patient's shoulders represented by the angles. There is no further information processing and the physician may use the data to evaluate patient's health. The designed systems measure head position with precision of $0,5^{\circ}$ (Hozman et al, 2008) in three planes (rotation -yaw, flexion-pitch and inclination-roll). Our experimental measurement of the head position was completed with measurement of subjective perception of vertical (SPV). The subject tried to align a needle to vertical position when peering into white sphere. Final angle of the needle was measured. The measured data shows that healthy subject holds his head aligned with physical coordinate system in the range of \pm 5 degrees for inclination. The set of data was measured on recruited volunteers. The results also predict that there is a correlation between values of inclination and SPV. 


\subsection{Eye and head movement analysis}

The Goal of our new designed methods is to use eye movements' detection together with the stimulation scene. Thanks to the special 3D HMD projection displays, we used Starburst algorithm for measuring in the IR spectrum and appropriate LED diode for illumination of the eye (Charfreitag et al, 2008). The Goal of the eye movements' measurement was to locate the centre of the pupil area (Stampe, 1993). We used a new system based on finding the contour line of the pupil of the eye. Finally, at the end, we can find the centre of the pupil from the resulting ellipse at the camera coordinate system i.e. shots, see Figure 8.

The second part was to use the headtracker to measure the head position. The first measured values were used as initial, i.e. zero and were used as correction for all subsequent values. The new systems provide direct information for physicians on the current position of the pupil centre represented by pixels or millimetres and patient's head represented by three angles, see Figure 9. There is no further information processing and the physician may use the data to evaluate patient's health. The head position was measured by modified 3D HMD (Z800 3DVisor) with precision of $1.0^{\circ}$ in three planes (Charfreitag et al, 2009). Thus, we can study the three dimensional motion of head defined by three angles - inclination, rotation and flexion/extension. By this method we can also study, analyze and measure eye and head movements continuously and simultaneously.

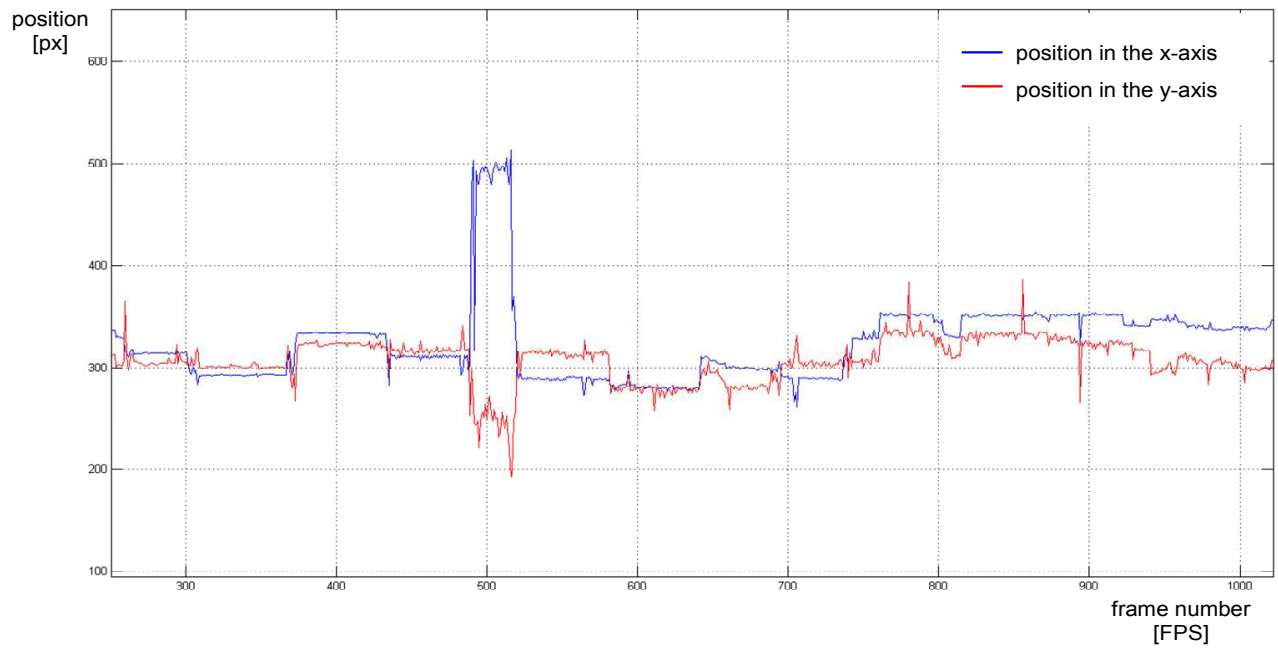

Fig. 8. Example of graph of pupil center movements 


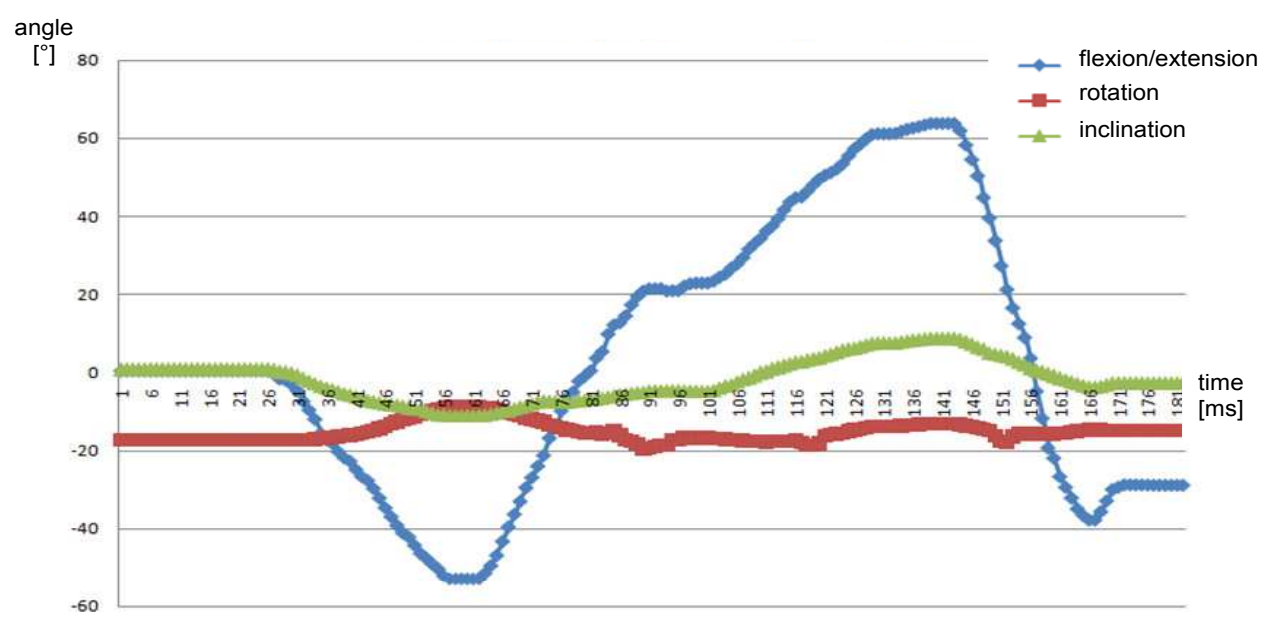

Fig. 9. Example of graph of head movements

\section{Conclusion}

In this chapter we have described related works and designed special equipment and measurement methods for very accurate evaluation of eye, head and shoulder position in neurological practice. Possible applications and perspectives for clinical practice are also described in the chapter.

We have described systems and sets of procedures for evaluation of the inclination, flexion and rotation of the head and the inclination and rotation of the shoulders with resolution and accuracy to $2^{\circ}$. This accuracy is the minimum accuracy required in clinical practice. The described ways of measuring and evaluating eye, head and shoulder positions could also be applied in other areas of medicine and science.

Our designed systems are based on cameras or/ possibly on gyro-accelerometer (inertial) sensors. The new two or three camera equipment designed to measure the head and shoulders positions is cheaper and more accurate than sophisticated systems which use accelerometers and magnetometers. The second advantage of our camera system over conventional and commercial systems such as Zebris motion analysis system (zebris Medical GmbH), LUKOtronic AS100/AS200 (Lukotronic Lutz-Kovacs-Electronics Oeg.) or sonoSens Monitor (sensomotion, Inc.) is that it can measure a patient without the influence of mechanical elements on patient's body segments or that the system allows direct detection of anatomical axes of patient's head and shoulders, which cannot be done when using current systems (Hozman et al, 2005). The systems based on two cameras have cameras placed on both sides (lateral profiles) or in front and above the patient. This is a very important advantage for medical doctors, because they can make various examinations which require open space in front of the face. Our systems based on combination of infrared cameras and inertial systems are also sufficient and more accurate and cheaper than commercial systems for broader use than just to analyze the position of the head and shoulders. 
The measurement results of mean values of the head position being (100 healthy controls): retro flexion $21.7^{\circ}$; inclination to the right $0.2^{\circ}$; head rotation to the left $1.7^{\circ}$. The rotation measurement has a greater error in comparison with the inclination and flexion/extension measurement (Kutilek \& Hozman, 2009).

We have also described related systems and designed system for monitoring eye movements. Our equipment designed for measurement of the eye and head movements is based on display units - specialized glasses with eMagin 3DVisor. We modified the specialized projection displays for neurological examination which can perform measurements using a variable set of visual stimuli and active head movements. The solution combines system for measurement of the eye movements and the head posture in the 3D space with 2D or 3D eye stimulation. We came to the conclusion that it is possible to join together the two important and closely related methods for the measurement of the human vestibular system.

A result of this study is the recommendation to use the video cameras with higher frequency (approximately $200 \mathrm{~Hz}$ ) for the measurement of eye movements and the head tracker with lower dynamic error (less than $0.3^{\circ} \mathrm{s}$ ) for the measurement of head position. The overall accuracy of our designed system could increase significantly because the accuracy of the method alone is in eights of degree per the ten measurements. This is the dynamic error due to the low-cost head tracker which needs long time to stabilise after the previous measurement.

Above described and designed ways of measuring eye, head and shoulder position and motion could also be applied in other areas of engineering, medicine and science. Our systems can be used anywhere to study the posture of a person.

\section{Acknowledgment}

The work presented here was carried out at the Czech Technical University in Prague, Faculty of Biomedical Engineering within the framework of research program No. MSM 6840770012 "Transdisciplinary Biomedical Engineering Research II" of the Czech Technical University, sponsored by the Ministry of Education, Youth and Sports of the Czech Republic.

\section{References}

Brandt, T., Cohen, B., Siebold, Ch. (2003). The Oculomotor and Vestibular Systems: Their Function and Disorders, Vol. 1004, Ann. N.Y. Acad. Sci.

Brandt T., Dieterich M. (1994). Vestibular Syndromes in the Roll Plane: Topographic Diagnosis from Brain Stem to Cortex, Annals of Neurology, Vol. 36, pp. 337-347.

Cerny R., Strohm K., Hozman J., Stoklasa J., Sturm D. (2006). Head in Space - Noninvasive Measurement of Head Posture, The 11th Danube Symposium - International Otorhinolaryngological Congress, Bled, pp. 39-42.

Cerny R., Hozman J., Charfreitag J., Kutílek P. (2009). Position of the head measured by digital photograph analysis, World Congress on Medical Physics and Biomedical 
Engineering, September 7 - 12, 2009, Munich, Germany [CD-ROM]. Berlin: Springer Science+Business Media , p. 562-565. ISBN 978-3-642-03881-5.

Charfreitag J., Hozman J., Cerny R. (2009). Measurement of eye and head position in neurological practice, World Congress on Medical Physics and Biomedical Engineering, September 7 - 12, 2009, Munich, Germany [CD-ROM]. Berlin: Springer Science+Business Media, p. 57-60. ISBN 978-3-642-03881-5.

Charfreitag J., Hozman J., Černý R. (2008). Specialized glasses - projection displays for neurology investigation, IFMBE Proceedings, Berlin: Springer, 2008, Vol. 1, p. 97-101. ISBN 978-3-540-89207-6.

Duchowski A. T., Medlin E., Cournia N. A., Murphy H. A., Gramopadhye A. K., Nair S. N., Vorah J., Melloy B. J. (2002). 3D Eye Movement Analysis, Behavior Research Methods, Instruments, \& Computers, Vol. 34, No. 4 , pp. 18.

Galardi G., Micera S., Carpaneto J., Scolari S., Gambini M., Dario P. (2003). Automated Assessment of Cervical Dystonia, Movement Disorders, Vol. 18, No. 11, pp. 13581367.

Gräf M., Droutsas K., Kaufmann H. (2001). Surgery for nystagmus related head turn: Kestenbaum procedure and artificial divergence, Graefes Arch Clin Exp Ophthalmol, Vol. 239, No. 5, pp. 334-341. doi:10.1007/s004170100270.

Harrison A., Wojtowicz G. (1996). Clinical Measurement of Head and Shoulder Posture Variables, The Journal of Orthopaedic \& Amp; Sports Physical Therapy (JOSPT), Vol. 23, pp. 353-361.

Hozman J., Sturm D., Stoklasa J. (2004). Measurement of Head Position in Neurological Practice, Biomedical Engineering, Zürich: Acta Press, p. 586-589. ISBN 0-88986379-2.

Hozman J., Kutílek P., Szabó Z., Krupička R., Jiřina M. (2008). Digital Wireless Craniocorpography with Sidelong Scanning by TV Fisheye Camera, IFMBE Proceedings, Berlin: Springer, Vol. 1, pp. 102-105. ISBN 978-3-540-892076.

Hozman J., Sturm D., Stoklasa J., Cerny R. (2005). Measurement of Postural Head Alignment in Neurological Practice, The 3rd European Medical and Biological Engineering Conference - EMBEC'05, Society of Biomedical Engineering and Medical Informatics of the Czech Medical Association JEP, Vol. 11, Prague, pp. 4229-4232.

Hozman J., Zanchi V., Cerny R., Marsalek P., Szabo Z. (2007). Precise Advanced Head Posture Measurement, The 3rd WSEAS International Conference on Remote Sensing (REMOTE'07), WSEAS Press, pp. 18-26.

Eui C. L., Kang R. P. (2007). A robust eye gaze tracking method based on a virtual eyeball model, Soul: Electronic Engineering of Yonsei University

Ferrario V., Sforza C., Germann D., Dalloca L., Miani A. (1994). Head Posture and Cephalometric Analyses: An Integrated Photographic/ Radiographic Technique, American Journal of Orthodontics \& Dentofacial Orthopedics, Vol. 106, pp. 257-264. 
Ferrario V., Sforza C., Tartaglia G., Barbini E., Michielon G. (1995). New Television Technique for Natural Head and Body Posture Analysis, Cranio, Vol. 13, pp. 247255.

Halmagyi M. G., Curthoys I. S., Brandt T., Dieterich M. (1991). Ocular Tilt Reaction: Clinical Sign of Vestibular Lesion, Acta Otolaryngologica, Suppl. 481, pp. 47-50.

Kutilek P., Hozman J. (2009). Non-contact method for measurement of head posture by two cameras and calibration means, The 8th Czech-Slovak Conference on Trends in Biomedical Engineering, Bratislava, pp. 51-54, ISBN 978-80-227-3105-8

Lee J. J., Park K. R., Kim J. H., (2003). Gaze detection system under HMD environment for user interface, Joint International conference ICANN/ICONIP 2003, Istanbul, pp. 512515.

Lee E. C., Park K. R. (2009). A robust eye gaze tracking method based on a virtual eyeball model, Machine Vision and Applications, Vol. 20, No. 5, pp. 319-337.

Li D. (2006) Low-cost eye-tracking for human computer interaction, Master's thesis, Iowa: Iowa State University

Meers S., Ward K., Piper I. (2006). Robust and Accurate Head-Pose Tracking Using a Single Camera, Mechatronics and Machine Vision in Practice, Berlin: Springer Science + Business Media, pp. 111-122, ISBN 978-3-540-74026-1.

Moore S., Curthoys I., Haslwanter T., Halmagyi M. (2006). Measuring Three-Dimensional Eye Position Using Image Processint - The VTM System, Sydney: Department of Psychology, University of Sydney

Murphy K., Preston Ch., Evans W. (1991). The Development of Instrumentation for the Dynamic Measurement of Changing Head Posture, American Journal of Orthodontics and Dentofacial Orthopedics, Vol. 99, No. 6, pp. 520-526.

Novak I., Campbell L., Boyce M., Fung V. S. (2010). Botulinum Toxin Assessment, Intervention and Aftercare for Cervical Dystonia and other Causes of Hypertonia of the Neck : International Consensus Statement, European Journal of Neurology, Vol. 17, Suppl. 2, pp. 94-108.

Nucci P., Kushner J. B., Serafino M, Orzalesi N. (2005). A Multi-Disciplinary Study of the Ocular, Orthopedic, and Neurologic Causes of Abnormal Head Postures in Children, American Journal of Ophthalmology, Vol. 140, pp. 65-68.

Palmgren P.J., Andreasson D., Eriksson M., Hägglund A. (2009). Cervicocephalic Kinesthetic Sensibility and Postural Balance in Patients with Nontraumatic Chronic Neck Pain - a Pilot Study, Chiropractic \& Osteopathy, Vol. 17, No. 6, doi:10.1186/1746-1340-17-6

Raine S., Twomey L.T. (1997). Head and Shoulder Posture Variations in 160 Asymptomatic Women and Men, Archives of Physical Medicine and Rehabilitation, Vol. 78, No. Nov., pp. 1215-1223.

Ruian L., Shijiu J., Xiaorong W. (2006). Single Camera Remote Eye Gaze Tracking Under Natural Head Movements, Tianjin: College of Physics and Electronic Information Science, Tianjin Normal University

Stampe D. M. (1993). Heuristic filtering and reliable calibration methods for video-based pupil tracking systems, Behavior Research Methods, Instruments and Computers, Vol. 25, No. 2, pp. 137-142. 
Young, J. D. (1988). Head Posture Measurement, Journal of Pediatric Ophthalmology and Strabismus, Vol. 25, No.2, pp. 86-89. 


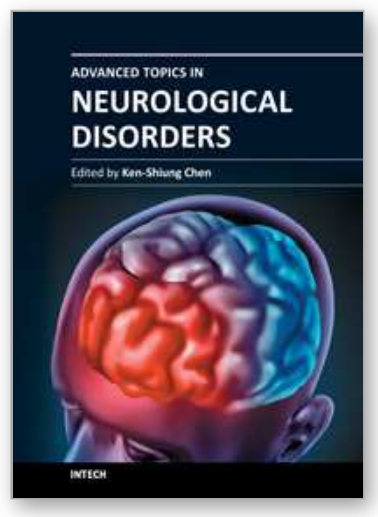

\author{
Advanced Topics in Neurological Disorders \\ Edited by Dr Ken-Shiung Chen
}

ISBN 978-953-51-0303-5

Hard cover, 242 pages

Publisher InTech

Published online 16, March, 2012

Published in print edition March, 2012

This book presents recent advances in the field of Neurological disorders research. It consists of 9 chapters encompassing a wide range of areas including bioengineering, stem cell transplantation, gene therapy, proteomic analysis, alternative treatment and neuropsychiatry analysis. It highlights the development of multiple discipline approaches in neurological researches. The book brings together leading researchers in neurological disorders and it presents an essential reference for researchers working in the neurological disorders, as well as for students and industrial users who are interested in current developments in neurological researches.

\title{
How to reference
}

In order to correctly reference this scholarly work, feel free to copy and paste the following:

Patrik Kutilek, Jiri Hozman, Rudolf Cerny and Jan Hejda (2012). Methods of Measurement and Evaluation of Eye, Head and Shoulders Position in Neurological Practice, Advanced Topics in Neurological Disorders, Dr Ken-Shiung Chen (Ed.), ISBN: 978-953-51-0303-5, InTech, Available from:

http://www.intechopen.com/books/advanced-topics-in-neurological-disorders/methods-of-measurement-andevaluation-of-eye-head-and-shoulders-position-in-neurological-practice

\section{INTECH}

open science | open minds

\section{InTech Europe}

University Campus STeP Ri

Slavka Krautzeka 83/A

51000 Rijeka, Croatia

Phone: +385 (51) 770447

Fax: +385 (51) 686166

www.intechopen.com

\section{InTech China}

Unit 405, Office Block, Hotel Equatorial Shanghai

No.65, Yan An Road (West), Shanghai, 200040, China

中国上海市延安西路65号上海国际贵都大饭店办公楼 405 单元

Phone: +86-21-62489820

Fax: +86-21-62489821 
(C) 2012 The Author(s). Licensee IntechOpen. This is an open access article distributed under the terms of the Creative Commons Attribution 3.0 License, which permits unrestricted use, distribution, and reproduction in any medium, provided the original work is properly cited. 